\title{
ISOMORPHISM OF HIERARCHICAL STRUCTURES
}

\author{
by \\ Charles Radin * and Lorenzo Sadun ** \\ Department of Mathematics, University of Texas, Austin, TX 78712
}

\begin{abstract}
We consider hierarchical structures such as Fibonacci sequences and Penrose tilings, and examine the consequences of different choices for the definition of isomorphism. In particular we discuss the role such a choice plays with regard to matching rules for such structures.
\end{abstract}

* Research supported in part by NSF Grant No. DMS-9531584 and Texas ARP Grant 003658-152

** Research supported in part by NSF Grant No. DMS-9626698 and Texas ARP Grant 003658-152 


\section{Introduction}

This paper concerns certain hierarchical tilings of Euclidean space, of which a well known example is a "kite \& dart" tiling of the plane [Gar]. Such tilings are noted for two properties: their unusual rotational symmetries ("ten-fold symmetry"), and the fact that their global structures derive from local ("matching") rules. We will examine the consequences of different choices for the definition of isomorphism between such structures. To be more specific, we assume that any tiling of interest is embedded in a compact metric space of tilings on which the connected component $G$ of the Euclidean group (or sometimes just its translation subgroup) acts in the natural way, and has a unique translation invariant Borel probability measure. We consider two specific notions of isomorphism: we say the tiling spaces $X$ and $Y$ are "topologically conjugate" if there is a homeomorphism between the spaces intertwining the actions of the group; and we say $X$ and $Y$ are "measureably conjugate" if there are subsets $Z_{X} \subset X$ and $Z_{Y} \subset Y$, of measure zero with respect to the unique translation invariant measure and invariant under the action of the group, and a bimeasurable map from $X / Z_{X}$ onto $Y / Z_{Y}$ intertwining the actions of the group. We investigate the consequences of assuming one or the other of these notions of isomorphism for such tilings.

In $[\mathrm{RaS}]$ we analyzed the rotational symmetry of such hierarchical structures, and found a way to associate a useful invariant for measureable conjugacy; the methods used would not have allowed for topological conjugacy, though perhaps other methods might. In this paper we analyze hierarchical structures from the point of view of their other main characteristic, the fact that their global structures derive from local rules, and here we show that topological conjugacy is definitely not as broadly applicable as measureable conjugacy.

The above approach to analyzing tilings is a natural outgrowth of the way symbolic sequences or arrays are studied, through embeddings in symbolic dynamical systems. The specific matter we consider for tilings, concerning local rules, is actually better understood in that older context. So we begin with more detailed descriptions of the relevant ideas within symbolic arrays.

One result of this paper is the sharpening of a remarkable result of Shahar Mozes [Moz]. Mozes showed that every substitution subshift with $\mathbb{Z}^{2}$ action, satisfying mild hypotheses, is measurably conjugate via an explicit construction to a uniquely ergodic subshift of finite type. (Such a result is commonly said to provide "matching rules" for the functions in the substitution subshift.) The map Mozes constructs is continuous (although not uniformly continuous) after exclusion of ap- 
propriate sets of measure zero. A natural question is whether such exclusion is an artifact of the proof or an essential feature of the theorem: Can Mozes' theorem be strengthened to give a topological conjugacy? We answer this question in the negative, exhibiting explicit substitution subshifts with $\mathbb{Z}^{2}$ action which are not topologically conjugate to any subshift of finite type.

After considering the simpler symbolic situation we explore the analogous questions for tiling dynamical systems with $\mathbb{R}^{d}$ actions. Mozes' proof can probably be extended without major difficulty to subshifts in higher dimensions and to tiling systems in which each polyhedron only appears, in any single tiling, in only finitely many orientations. However, more general tiling systems, such as the pinwheel in $\mathbb{R}^{2}$ [Ra1] and quaquaversal tilings in $\mathbb{R}^{3}[\mathrm{CoR}]$, require a significant extension of the proof in [Moz]. This was done for the pinwheel in [Ra1] and then in general in $[\mathrm{GoS}]$. In each case a measureable conjugacy is constructed, but the map is only continuous after exclusion of certain sets of measure zero. We demonstrate, as with subshifts, that these theorems cannot be strengthened to the topological category: there exist substitution tiling systems that are measureably conjugate to finite type tiling systems but which are not topologically conjugate to any finite type tiling system.

In both settings, with subshifts and tilings, we give conditions, easily satisfied in natural examples, for which such a topological conjugacy is not possible. However, the two constructions are somewhat different for there are important properties of subshifts whose natural analogues do not hold for tilings. For instance, for subshifts it is well known that every topological conjugacy is a sliding block code while for tilings we show this to be false.

This last point is both surprising and profound. The continuous nature of the $\mathbb{R}^{d}$ actions allows for two kinds of small changes to a tiling. Tiles may be changed near infinity, or a small Euclidean motion may be applied to the entire tiling. Neither notion is topologically invariant since, as we show, a topological conjugacy can encode pattern information from distant regions as small Euclidean motions near the origin. This mechanism, which has no analogue in the theory of subshifts (where the translation group acts discontinuously), indicates that the nature of topological conjugacies of tilings is much subtler than topological conjugacy of subshifts.

In section 1 we discuss subshifts and show that many substitution subshifts are not topologically conjugate to finite type subshifts. In section 2 we explore topological conjugacy in tiling systems, giving an example of a conjugacy that is not a sliding block code. This example also demonstrates that local finiteness, a 
property automatic for subshifts, is not a topological invariant for tilings. In section 3 we return to the question of Mozes' theorem, only now for tilings, and show there exist subsitution tilings that are measureably conjugate, but not topologically conjugate, to finite type tilings.

\section{Notation and results for subshifts}

For $n \geq 0$ and finite abstract alphabet $\mathcal{A}$ (always assumed nonempty) let $\prod_{n}$ be the restriction of functions in $\mathcal{A}^{\mathbb{Z}^{d}}$ onto $[-n, n]^{d}$. Let $\sigma_{\mathcal{A}}^{j}$ denote the shift by $j \in \mathbb{Z}^{d}$ on (any subshift of) $\mathcal{A}^{\mathbb{Z}^{d}}:\left(\sigma_{\mathcal{A}}^{j} x\right)_{k}=x_{k+j}$. For each $n \geq 1$ define the set

$$
X_{n}=\left\{x \in \mathcal{A}^{\mathbb{Z}^{d}}: \prod_{n}\left[\sigma_{\mathcal{A}}^{j} x\right] \in \prod_{n}[X] \text { for all } j \in \mathbb{Z}^{d}\right\} .
$$

That is, $X_{n}$ is the set of functions that, restricted to cubes of size $n$, look like elements of $X$. We say the subshift $X$ is "of finite type" if $X=X_{n}$ for some $n \geq 1$.

Given any second alphabet $\mathcal{B}$ and a "block map" $\Phi: \prod_{n}(X) \longrightarrow \mathcal{B}$ we can define the "sliding block code (of code size $n$ )" $\phi: X \rightarrow Y$ by $\phi(x)_{j}=\Phi\left(\prod_{n}\left[\sigma_{\mathcal{A}}^{j}(x)\right]\right.$ ). Note that $\phi$ is continuous and intertwines the shifts on $X$ and $\phi(X) \subset \mathcal{B}^{\mathbb{Z}^{d}}$; if it is also invertible it is a topological conjugacy. An equivalent description of a sliding block code of size $n$ is a map $X \rightarrow Y$, intertwining the $\mathbb{Z}^{d}$ actions, such that, whenever $\prod_{n} x=\prod_{n} x^{\prime}$, then $\prod_{0} \phi(x)=\prod_{0} \phi\left(x^{\prime}\right)$.

Theorem 1. (Curtis-Lyndon-Hedlund; [LiM]). Any topological conjugacy $\phi$ between $\mathbb{Z}^{d}$ subshifts $X$ and $Y$ is a sliding block code.

Proof: Let $d_{X}$ and $d_{Y}$ be the metrics on $X$ and $Y$, respectively. Since $X$ and $Y$ are compact metric spaces, any continuous map between them is uniformly continuous. We can therefore pick constants $\epsilon, \delta$ and $n$ such that $\prod_{n} x=\prod_{n} x^{\prime}$ implies $d_{X}\left(x, x^{\prime}\right)<\delta$, which implies $d_{Y}\left(\phi(x), \phi\left(x^{\prime}\right)\right)<\epsilon$, which implies $\prod_{0} \phi(x)=\prod_{0} \phi\left(x^{\prime}\right)$.

The following theorem seems to be widely known but does not appear to be in the literature. The proof we give was shown to us by Klaus Schmidt.

Theorem 2. Assume the $\mathbb{Z}^{d}$ subshift $X$ is of finite type and topologically conjugate to the subshift $Y$. Then $Y$ is of finite type.

Proof. Assume $X=X_{n}$ and $\phi: Y \longrightarrow X$ is the topological conjugacy. By Theorem 1 both $\phi$ and $\phi^{-1}$ are sliding block codes, say of size $m$ and $m^{\prime}$, so there exists a block map $\Phi: \prod_{m}[Y] \rightarrow \mathcal{A}$ such that $\phi(y)_{j}=\Phi\left(\prod_{m}\left[\sigma_{\mathcal{B}}^{j}(y)\right]\right)$. For any $p \geq m$ we can extend $\phi$ to $\phi^{\prime}: Y_{p} \rightarrow X^{\mathcal{A}}$ by this rule. Notice that the image of $\phi^{\prime}$ is in $X_{p-m}$, 
so, for $p \geq m+n, \phi^{\prime} \operatorname{maps} Y_{p}$ to $X$.

Now fix $p=\max \left(m+m^{\prime}, m+n\right)$ and let $\rho=\phi^{-1} \circ \phi^{\prime}: Y_{p} \longrightarrow Y$. Since $\rho$ is the product of sliding block codes of size $m$ and $m^{\prime}$, it is itself a sliding block code of size $k=m+m^{\prime} . \rho$ is the identity when restricted to $Y$; since $k \leq p, \prod_{k} Y_{p}=\prod_{k} Y$, so the map $\rho$ is the identity on $Y_{p}$, implying $Y_{p}=Y$.

We next define substitution subshifts and show that certain substitution subshifts are not of finite type. By Theorem 2 they cannot be topologically conjugate to subshifts of finite type, but by Mozes' theorem they are measureably conjugate to subshifts of finite type.

We begin in dimension 1. Given an alphabet $\mathcal{A}$ we define the set of "words" as $\mathcal{W}=\cup_{n \geq 1} \mathcal{A}^{n}$. We assume given a "substitution function" $\psi: \mathcal{A} \rightarrow \mathcal{W}$ such that $\psi(a) \in \cup_{n \geq 2} \mathcal{A}^{n}$ for some $a \in \mathcal{A}$. A word of the form $\psi^{n}(a)$ is said to be a "letter of level $n$ " for any $n \geq 0$, and we denote by $\mathcal{W}^{\psi}$ the set of all such words. Finally, we define the substitution subshift associated with the substitution $\psi$ as

$\left\{x \in \mathcal{A}^{\mathbb{Z}} \mid\right.$ For all $j \in \mathbb{Z}, k \geq 0$ there exists $w \in \mathcal{W}^{\psi}$ s.t. $\left.\left(x_{j}, x_{j+1}, \cdots, x_{j+k}\right) \subseteq w\right\}$.

It is a simple fact that every function in such a subshift can simultaneously be considered a function with values in $\psi^{n}(\mathcal{A})$ for each value of $n \geq 0$. In interesting cases functions in a substitution subshift can be considered functions with values in letters of each higher level in only one way; such subshifts are said to be "uniquely derivable". (This is the origin of the term "hierarchical" for these structures. The same hierarchical phenomenon exists for tilings, and in a manner easier to understand, which we illustrate in Figure 2.)

The above has a straightforward generalization to $\mathbb{Z}^{d}$ subshifts as long as the images of the substitution function fit together geometrically, so that one can iterate the substitution. This is automatic for $\mathbb{Z}^{d}$ substitution subshifts that are products of $\mathbb{Z}$ substitution subshifts, which we illustrate by an example below.

Corollary 1. If a $\mathbb{Z}^{2}$ substitution subshift $X$ contains a function $x$ such that $x_{j, k}=x_{j, k+1}=x_{j+1, k}=x_{j+1, k+1}$ for some $(j, k) \in \mathbb{Z}^{2}$, and $X$ is topologically conjugate to a finite type subshift, then $X$ contains a periodic function.

Proof. The condition on $x$ defines a word $C \in \mathcal{A}^{[0,1]^{2}}$. Consider the large words $W \in$ $\mathcal{A}^{[0, N-1] \times[0, M-1]}$ produced when the substitution is applied repeatedly to $C$, and consider the periodic function $p$ defined by the condition $\left(\sigma_{\mathcal{A}}^{N j, M k} p\right)_{[0, N-1] \times[0, M-1]}=$ $W$ for all $j, k \in \mathbb{Z}$. Since $X$ is topologically conjugate to a subshift of finite type, by Theorem 2 there is some $n \geq 1$ such that $X=X_{n}$. Taking $W$ large enough it 
follows that $X$ contains the periodic function $p$.

Corollary 2. There exists a $\mathbb{Z}^{2}$ substitution subshift that is measureably conjugate to a finite type subshift, but is not topologically conjugate to any finite type subshift.

Proof. Consider the uniquely derivable symbolic Fibonacci substitution defined, using the alphabet $\mathcal{A}=\{a, b\}$, by the substitution function $\psi(a)=b, \psi(b)=a b$; let $F(1)$ be the strictly ergodic substitution subshift thus determined. Let $F(2) \subset$ $\{\mathcal{A} \times \mathcal{A}\}^{\mathbb{Z}^{2}}$ be the substitution subshift which is the product of $F(1)$ with itself:

$$
\begin{aligned}
& \psi_{2}(a \times a)=(b \times b) ; \quad \psi_{2}(b \times a)=\left(\begin{array}{ll}
a \times b & b \times b) ;
\end{array}\right. \\
& \psi_{2}(a \times b)=\left(\begin{array}{c}
b \times b \\
b \times a
\end{array}\right) ; \quad \psi_{2}(b \times b)=\left(\begin{array}{cc}
a \times b & b \times b \\
a \times a & b \times a
\end{array}\right) .
\end{aligned}
$$

It follows [Moz] that $F(2)$ is uniquely derivable. Mozes' theorem states that under rather general conditions (satisfied by $F(2)$ ) a $\mathbb{Z}^{2}$ substitution subshift $X$ which is uniquely derivable is measurably conjugate to some finite type subshift, so $F(2)$ is measurably conjugate to a finite type subshift.

A simple calculation, however, shows that $\psi_{2}^{3}(b \times b)$, and hence all functions in $F(2)$, contain the block $\left(\begin{array}{cc}b \times b & b \times b \\ b \times b & b \times b\end{array}\right)$, and a standard argument ([Ra2], [Ra3]) using the unique derivability of $F(2)$ implies that $F(2)$ contains no periodic functions. Corollary 1 then implies that $F(2)$ is not topologically conjugate to any finite type subshift.

\section{Conjugacies of Fibonacci Tilings}

Now we consider "tiling systems". Given a finite collection $\mathcal{A}$ of polyhedra in $d$ dimensions, we define $X^{\mathcal{A}}$ as the space of all tilings of Euclidean $d$-space by congruent copies of elements of $\mathcal{A}$. (We assume $X^{\mathcal{A}}$ is nonempty.) We put a metric $d(\cdot, \cdot)$ on $X^{\mathcal{A}}$ as follows.

$$
d(x, y) \equiv \sup _{n} \frac{1}{n} m_{H}\left[B_{n}(\partial x), B_{n}(\partial y)\right]
$$

where $B_{n}(\partial x)$ denotes the intersection of two sets: the closed ball $B_{n}$ of radius $n$ centered at the origin of the Euclidean space and the union $\partial x$ of the boundaries $\partial a$ of all tiles $a$ in $x . m_{H}$ is the Hausdorff metric on compact sets defined as follows. Given two compact subsets $A$ and $B$ of $\mathbb{R}^{m}, m_{H}[A, B]=\max \{\tilde{d}(A, B), \tilde{d}(B, A)\}$, where

$$
\tilde{d}(A, B)=\sup _{a \in A} \inf _{b \in B}\|a-b\|,
$$

with $\|w\|$ denoting the usual Euclidean norm of $w$. It is not hard to show that with this metric $X^{\mathcal{A}}$ is compact and that the natural representation of the connected 
component of the Euclidean group on $X^{\mathcal{A}}$ is continuous. Finally, let $\hat{G}$ be either the the group of translations of $\mathbb{R}^{d}$ or the connected component of the Euclidean group. A tiling system is a closed $\hat{G}$-invariant subset of $X^{\mathcal{A}}$.

Two important properties of tiling systems are "local finiteness" and "finite type". For each $R>0$ and polyhedron $a \in \mathcal{A}$ appearing in the tiling $x$ of $X$ consider the set $H(R, x, a)$ of polyhedra in $x$ which intersect the open ball of radius $R$ centered at the center of mass of $a$. Define $H(R, X)$ to be the union of all such "neighborhoods of radius $R$ " for all $a \in x$ and $x \in X$. A tiling system $X$ is "locally finite" if, for every $R>0$, the set $H(R, X)$ is finite up to the action of $\hat{G}$. In practice, tiling systems are almost always assumed to be locally finite.

Now, in analogy to the subshifts $X_{n}$, we define $X_{R}$ to be the set of all $x$ in $X^{\mathcal{A}}$ such that $x$ only has neighborhoods of radius $R$ which are in $H(R, X)$. A tiling system $X$ is said to be "of finite type" if $X=X_{R}$ for some $R>0$.

We will be considering the analogue among tiling systems of the substitution subshifts. Traditionally such substitution tiling systems are defined through a substitution function as for subshifts, whereby for each letter (polyhedron) there is given a way to decompose it into polyhedra similar to those in the alphabet, all smaller by some factor $\gamma<1$, so that following the decomposition by a stretch about the origin by $1 / \gamma$ one associates to each letter of $\mathcal{A}$ a letter of level 1 , and so on. For example, Figure 1 shows a substitution for a "chair" tile, in this case with $\gamma=1 / 2$, and Figure 2 shows the hierarchical structure in a piece of a chair tiling. Applying the substitution to all tiles in a tiling gives an automorphism $\psi: X \rightarrow X$. Notice that $\psi$ commutes with rotations about the origin and that $\psi \circ \sigma^{\alpha}=\sigma^{\alpha / \gamma} \circ \psi$, where $\sigma^{\alpha}$ is a translation by $\alpha \in \mathbb{R}^{d}$.

We use this last property to define a generalized notion of substitution tiling system. For our purposes, a substitution tiling system is a tiling system with an automorphism $\psi$ and a constant $\gamma<1$ such that $\psi$ commutes with any rotations about the origin in $\hat{G}$ and such that $\psi \circ \sigma^{\alpha}=\sigma^{\alpha / \gamma} \circ \psi$ for all $\alpha \in \mathbb{R}^{d}$.

A simple example of a substitution tiling is the standard Fibonacci tiling in one dimension. In this tiling the alphabet consists of two tiles (intervals), $A$ and $B$, of length $|A|=1$ and $|B|=\tau=[1+\sqrt{5}] / 2$. The substitution consists of expanding about the origin by a factor of $\tau$, and then replacing each expanded $A$ (of size $\tau$ ) with a $B$, and replacing each expanded $B$ (of size $\tau^{2}=1+\tau$ ) with an $A$ and a $B$. The resulting space of tilings is precisely the set of tilings by $A$ 's and $B$ 's arrayed in the sequence of the Fibonacci subshift $F(1)$.

This last characterization allows us to define general Fibonacci tilings, based 
on any two tiles $A$ and $B$, as the set of tilings in which the pattern of $A$ 's and $B$ 's is the same as a sequence in the Fibonacci subshift. If $|B| \neq \tau|A|$ this is not a substitution tiling in the traditional sense, as the substitution $A \rightarrow B, B \rightarrow A B$ is not an expansion by a constant factor. However, it is a substituion tiling system in the extended sense of this paper.

Theorem 3. Let $X$ and $Y$ be Fibonacci tiling systems, with tiles $A_{X}, B_{X}$ and $A_{Y}, B_{Y}$, respectively. If $\left|A_{X}\right|+\tau\left|B_{X}\right|=\left|A_{Y}\right|+\tau\left|B_{Y}\right|$ then $X$ and $Y$ are topologically conjugate. Moreover, the conjugacy is not a sliding block code.

Proof: We define a map $\phi: X \longrightarrow Y$ as follows. A tiling $x \in X$ defines an element of $F(1)$ (up to translation), which in turn defines a tiling $y \in Y$, up to translation. To fix the translation we pick a tile $a \in x$. This tile $a$ is a letter of level 0 , which sits inside a letter $a_{1}$ of level 1 , which sits inside a letter $a_{2}$ of level 2 , and so on. We approximate the placement of the tiles in $y$ by lining up the middle of the letter in $y$ that corresponds to $a_{n}$ with the middle of $a_{n}$. Since $\left|A_{X}\right|+\tau\left|B_{X}\right|=\left|A_{Y}\right|+\tau\left|B_{Y}\right|$, the letters of level $n$ in the two tilings differ in size by a constant times $\tau^{-n}$. The adjustment in translation between the $n$th approximant and the $n+1$ st is then exponentially small, and we can take the limit as $n \rightarrow \infty$ to obtain $\phi(x)$. Loosely speaking, the map $\phi$ lines up the corresponding letters of infinite level.

We must show that this map is well defined, in that it does not depend on the choice of tile $a$. If $a^{\prime} \in x$ is another tile, then there are two possibilities. The more common possibility is that there is a level $n$ at which $a_{n}=a_{n}^{\prime}$, so the approximants to $\phi(x)$ based on $a^{\prime}$ are eventually the same as the approximants based on $a$. Alternatively, there may be a point between $a$ and $a^{\prime}$ which is the endpoint of letters of arbitrarily high level. It is not hard to check that, working from either $a$ or $a^{\prime}, \phi$ aligns this special point in $x$ with the corresponding special point in $\phi(x)$.

The map $\phi$ is clearly a bijection which commutes with translations. It is continuous since a small change to a tiling $x$, either via a small translation or by changing the tiles near infinity, results is a small change to $\phi(x)$. A small translation in $x$ turns into a small translation in $\phi(x)$, while changing the distant tiles of $x$ changes the distant tiles of $\phi(x)$, and also causes a small translation.

This last point means that $\phi$ is not a sliding block code. If two tilings $x$ and $x^{\prime}$ agree exactly on a large neighborhood of the origin but disagree near infinity, then the sequence of tiles of $\phi(x)$ and $\phi\left(x^{\prime}\right)$ will agree near the origin, but the placement of the tiles will differ by a small translation. Thus there is no neighborhood of the origin where $\phi(x)$ and $\phi\left(x^{\prime}\right)$ agree exactly. 
There are two special Fibonacci tilings. One is the standard Fibonacci tiling with $|B|=\tau|A|$. The other has $|B|=|A|$, and thus closely resembles the Fibonacci subshift $F(1)$. By Theorem 3 any Fibonacci tiling is conjugate to each of these. In particular,

Corollary 3. Every Fibonacci tiling system is a substitution tiling system.

Proof: Every Fibonacci tiling system is topologically conjugate to a Fibonacci system $X$ with $\left|B_{X}\right|=\tau\left|A_{X}\right|$. As noted earlier, $X$ is a substitution system with automorphism $\psi_{X}$ and scale factor $\gamma=1 / \tau$. If $Y$ is another Fibonacci tiling system and $\phi: X \longrightarrow Y$ is the conjugacy of Theorem 3, then $\psi_{Y}=\phi \circ \psi_{X} \circ \phi^{-1}$ is a substitution automorphism on $Y$.

Unlike a sliding block code the domain of a topological conjugacy $\phi: X \longrightarrow Y$ of tiling systems cannot always be extended to $X_{R}$ for $R$ sufficiently large. Suppose for example that $X$ and $Y$ are Fibonacci tiling systems with $\left|A_{X}\right|=1,\left|B_{X}\right|=\tau$, $\left|A_{Y}\right|=2$ and $\left|B_{Y}\right|=1$. For any $R$ the system $X_{R}$ contains periodic tilings, whose period $T$ is an integral linear combination of 1 and $\tau$. Since the tiles of $\mathcal{A}_{Y}=\left\{A_{Y}, B_{Y}\right\}$ have integer length, there are no periodic tilings in $X^{\mathcal{A}_{Y}}$ with period $T$, and so there are no maps $X_{R} \longrightarrow X^{\mathcal{A}_{Y}}$ that intertwine translations.

Since topological conjugacies of tilings are more general than sliding block codes, topological invariants of subshifts are not automatically topological invariants of tilings. For example,

Theorem 4. There exist topologically conjugate tiling systems $X$ and $Y$ such that $X$ is locally finite and $Y$ is not.

Proof: We work in 2 dimensions, with $\hat{G}$ being the translation group. Given two square tiles $A_{X}$ and $B_{X}$ of unit size, with edges parallel to the coordinate axes, let $X$ be the set of all tilings such that the tiles meet full edge to full edge, and such that each (horizontal) row of the tiling is a (1 dimensional) Fibonacci tiling. Let $\mathcal{A}_{Y}=\left\{A_{Y}, B_{Y}\right\}$, where $A_{Y}$ is a rectangle of height 1 and width $\tau$ and $B_{Y}$ is a rectangle of height 1 and width $\tau-1$. Let $\phi$ be the conjugacy of Theorem 3, taking the 1 dimensional Fibonacci tiling with tile sizes 1 and 1 to the 1 dimensional Fibonacci tiling with sizes $\tau$ and $\tau-1$. This map is naturally extended to a map from rows of the tiling $X$ to Fibonacci-like rows made up of the tiles of $\mathcal{A}_{Y}$. Applying this to every row of a tiling $x \in X$ defines an extended map (also called $\phi$ ) from $X$ into $X^{\mathcal{A}_{Y}}$. Let $Y$ be the image of this map. Near the origin (or any other point), the rows of a tiling $y \in Y$ will appear shifted relative to one another. Since there are an infinite number of ways in which this shift can occur, $Y$ is not locally finite. 


\section{Conjugacies of tiling systems of finite type}

In this section tiling systems are assumed to be locally finite unless stated otherwise.

A natural question, in the light of Mozes' theorem, is whether Theorem 2 can be extended to the category of tiling systems. Certainly the proof breaks down, as conjugacies of tilings are not necessarily sliding block codes and need not extend to spaces $X_{R}$. However, there is hope that the statement of the theorem remains true.

In this section we prove a somewhat weaker result, the analogue of Corollary 1 , thereby showing that there exist tiling systems that are measurably conjugate to finite type systems but not topologically conjugate. The key is showing that topological conjugacies, while not sliding block codes, do preserve some useful geometrical information. One can model a topological conjugacy $\phi: X \rightarrow Y$ between tiling systems $X$ and $Y$ - with metrics $d_{X}(\cdot, \cdot)$ and $d_{Y}(\cdot, \cdot)$ and alphabets $\mathcal{A}$ and $\mathcal{B}$ - by something close to a sliding block code, as follows.

First we note that $\phi$ is uniformly continuous. So given $\epsilon>0$ there is some $\delta>0$ such that $d_{Y}\left[\phi(x), \phi\left(x^{\prime}\right)\right]<\epsilon$ provided $d_{X}\left[x, x^{\prime}\right]<\delta$. Now it is known $[\mathrm{RaW}]$ that given any $\chi>0$ there is an $\epsilon>0$ such that if $d_{Y}\left[y, y^{\prime}\right]<\epsilon$ it follows that there is a rigid motion $t$ within distance $\chi$ of the identity in $\hat{G}$ such that the tilings $y$ and $\sigma_{\mathcal{B}}^{t}\left(y^{\prime}\right)$ "agree to distance 1 around the origin", in the sense that they match perfectly in their polyhedra which intersect the ball of radius 1 centered at the origin. Furthermore there is some $R>0$ such that if tilings $x$ and $x^{\prime}$ in $X$ agree to distance $R$ around the origin then $d_{X}\left[x, x^{\prime}\right]<\delta$ and so the tilings $\phi(x)$ and $\sigma_{\mathcal{B}}^{t}\left[\phi\left(x^{\prime}\right)\right]$ agree to distance 1 around the origin for some $t \in \hat{G}$ of distance less than $\chi$ from the identity. We have proven the following theorem.

Theorem 5. Let $\phi$ be a topological conjugacy of the locally finite tiling system $X$ in $\mathbb{R}^{d}$ onto the locally finite $\mathbb{R}^{d}$ tiling system $Y$. Given $\epsilon>0$ there is some $R_{\phi}(\epsilon)>0$ such that if tilings $x$ and $x^{\prime}$ in $X$ agree to distance $R_{\phi}(\epsilon)$ around the origin then the tilings $\phi(x)$ and $\sigma_{\mathcal{B}}^{t}\left[\phi\left(x^{\prime}\right)\right]$ agree to distance 1 around the origin for some rigid motion $t$ of distance less than $\epsilon$ from the identity in $\hat{G}$.

For any bounded connected subset $P$ of $\mathbb{R}^{d}, R>0$, and tiling $x$, let $P^{R}$ be the union of open balls of radius $R$ which intersect $P$.

Corollary 4. Let $\phi: X \rightarrow Y$ be a topological conjugacy of locally finite tiling systems in $\mathbb{R}^{d}$. Then given $\epsilon>0$ and a bounded connected subset $P$ of $\mathbb{R}^{d}$ there exists $\mathbb{R}_{\phi}(\epsilon)>0$ such that if tilings $x$ and $x^{\prime}$ in $X$ agree on $P^{R_{\phi}(\epsilon)}$ there is a rigid motion $t$ of distance less than $\epsilon$ from the identity in $\hat{G}$ such that $\phi(x)$ and $\sigma_{\mathcal{B}}^{t}\left[\phi\left(x^{\prime}\right)\right]$ 
agree on $P$.

Proof. The proof follows immediately by applying Theorem 5 to all the balls of radius $R_{\phi}(\epsilon)$ which intersect $P$, and noting that their overlapping regions lead to consistency requirements in $Y$.

We say that a tiling $x$ in an $\mathbb{R}^{2}$ tiling system $X$ with alphabet $\mathcal{A}$ has a "periodic frame" if there is some pair $\{s, t\}$ of linearly independent translations for which $\sigma_{\mathcal{A}}^{a s+b t} x$ and $\sigma_{\mathcal{A}}^{a s+(b+1) t} x$ agree to distance 1 about the origin for all $0 \leq a \leq 1$ and $|b| \leq 1 / 8$, and also $\sigma_{\mathcal{A}}^{a s+b t} x$ and $\sigma_{\mathcal{A}}^{(a+1) s+b t} x$ agree to distance 1 about the origin for all $0 \leq b \leq 1$ and $|a| \leq 1 / 8$.

Theorem 6. If an $\mathbb{R}^{2}$ substitution tiling system $X$ contains a tiling with a periodic frame, and $X$ is topologically conjugate to a finite type tiling system, then $X$ contains a periodic tiling.

Proof. Assume $\epsilon>0$ is given and the frame is described by with the defining notation above. By using the substitution repeatedly if necessary we can assume without loss of generality that $\min \{\| m s+n t||, m, n \in \mathbb{Z}\}$ is larger than any given number. So we can assume $x$ and $\sigma_{\mathcal{A}}^{t} x$ agree on $P_{1}^{R_{\phi}(\epsilon)}$ where $P_{1}=\{a s+b t: 0 \leq$ $a \leq 1,|b| \leq 1 / 8\}$ and that $x$ and $\sigma_{\mathcal{A}}^{s} x$ agree on $P_{2}^{R_{\phi}(\epsilon)}$ where $P_{2}=\{a s+b t: 0 \leq b \leq$ $1,|a| \leq 1 / 8\}$. Applying Corollary 2 we see that $\phi(x)$ and $\sigma_{\mathcal{B}}^{t+\tau} \phi(x)$ agree on $P_{1}$ and that $\phi(x)$ and $\sigma_{\mathcal{B}}^{s+\tau^{\prime}} \phi(x)$ agree on $P_{2}$ for rigid motions $\tau$ and $\tau^{\prime}$ within distance $\epsilon$ of the identity of $\hat{G}$. Note that because of the overlap between them, it follows that $\tau$ and $\tau^{\prime}$ are pure translations and so the central lines of $P_{1}, \sigma_{\mathcal{B}}^{t+\tau} P_{1}, P_{2}$ and $\sigma_{\mathcal{B}}^{s+\tau^{\prime}} P_{2}$ form a parallelogram. And since $Y$ is of finite type this implies that it contains a periodic tiling. But then so does $X$.

Remark: At the end of section 1 we showed that the subshift with $\mathbb{Z}^{2}$ action made as a product of the Fibonacci sequences with itself could not be topologically conjugate to a finite type subshift. Theorem 6 allows us to apply the same argument to tilings. Starting with any Fibonacci tiling system we can define the product tiling system with $\mathbb{R}^{2}$ action and show that it contains a tiling with a periodic frame, and therefore cannot be topologically conjugate to a finite type tiling system since it does not contain a periodic tiling.

\section{Conclusion}

The best known tiling dynamical system is that of the Penrose kites \& darts [Ra2, Ra3]. By this one refers to both a substitution system and a finite type system, which are in fact topologically conjugate. The fact that the conjugacy is topological has lead to interesting work in noncommutative topology [AnP, Con]. 
To a large extent the interest in substitution tiling systems is due to the fact that they are conjugate to finite type systems. Theorem 6 shows that, when dealing with substitution tilings and their conjugacy to finite type tilings, for reasonable generality one cannot consider the tilings well defined up to topological conjugacy though of course measurable conjugacy is sufficient. One issue we have not resolved is whether or not the notion of finite type is itself a topological invariant among tiling systems.

\section{Acknowledgements}

We thank Klaus Schmidt for showing us the proof of Theorem 2.

\section{REFERENCES}

[AnP] J. Anderson and I. Putnam, Topological invariants for substitution tilings and their associated $\mathrm{C}^{\star}$-algebras, Erg. Thy. Dyn. Syst., to appear.

[Con] A. Connes, Noncommutative Geometry, Academic Press, San Diego, 1994.

[CoR] J.H. Conway and C. Radin, Quaquaversal tilings and rotations, Invent. math. 132 (1998), 179-188.

[Gar] M. Gardner, Extraordinary nonperiodic tiling that enriches the theory of tiles, Sci. Am. (USA) (December 1977), 110-119.

[GoS] C. Goodman-Strauss, Matching rules and substitution tilings, Ann. of Math. 147 (1998), 181-223.

[LiM] D. Lind and B. Marcus, An Introduction to Symbolic Dynamics and Coding, Cambridge University Press, Cambridge, 1995.

[Moz] S. Mozes, Tilings, substitution systems and dynamical systems generated by them, J. d'Analyse Math. 53 (1989), 139-186.

[Ra1] C. Radin, The pinwheel tilings of the plane, Ann. of Math. 139 (1994) 661-702.

[Ra2] C. Radin, Miles of Tiles, Ergodic theory of $\mathbb{Z}^{d}$-actions, London Math. Soc. Lecture Notes Ser. 228 Cambridge University Press, Cambridge, 1996, pp. 237-258. 
[Ra3] C. Radin, Aperiodic Tiling. Available from the electronic preprint archive mp_arc@math.utexas.edu as 98-232.

[RaS] C. Radin and L. Sadun, An algebraic invariant of substitution tiling systems, Geometriae Dedicata (to appear).

[RaW] C. Radin and M. Wolff, Space tilings and local isomorphism, Geometriae Dedicata 42 (1992), 355-360. 

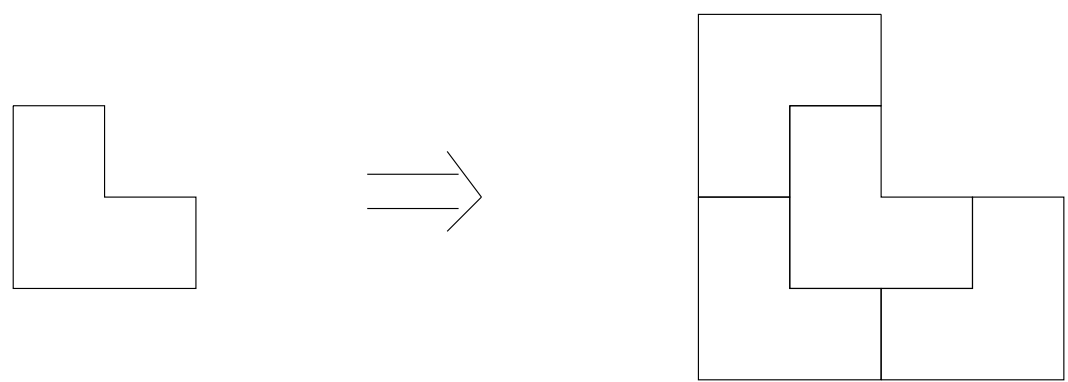

Fig. 1. The chair substitution 


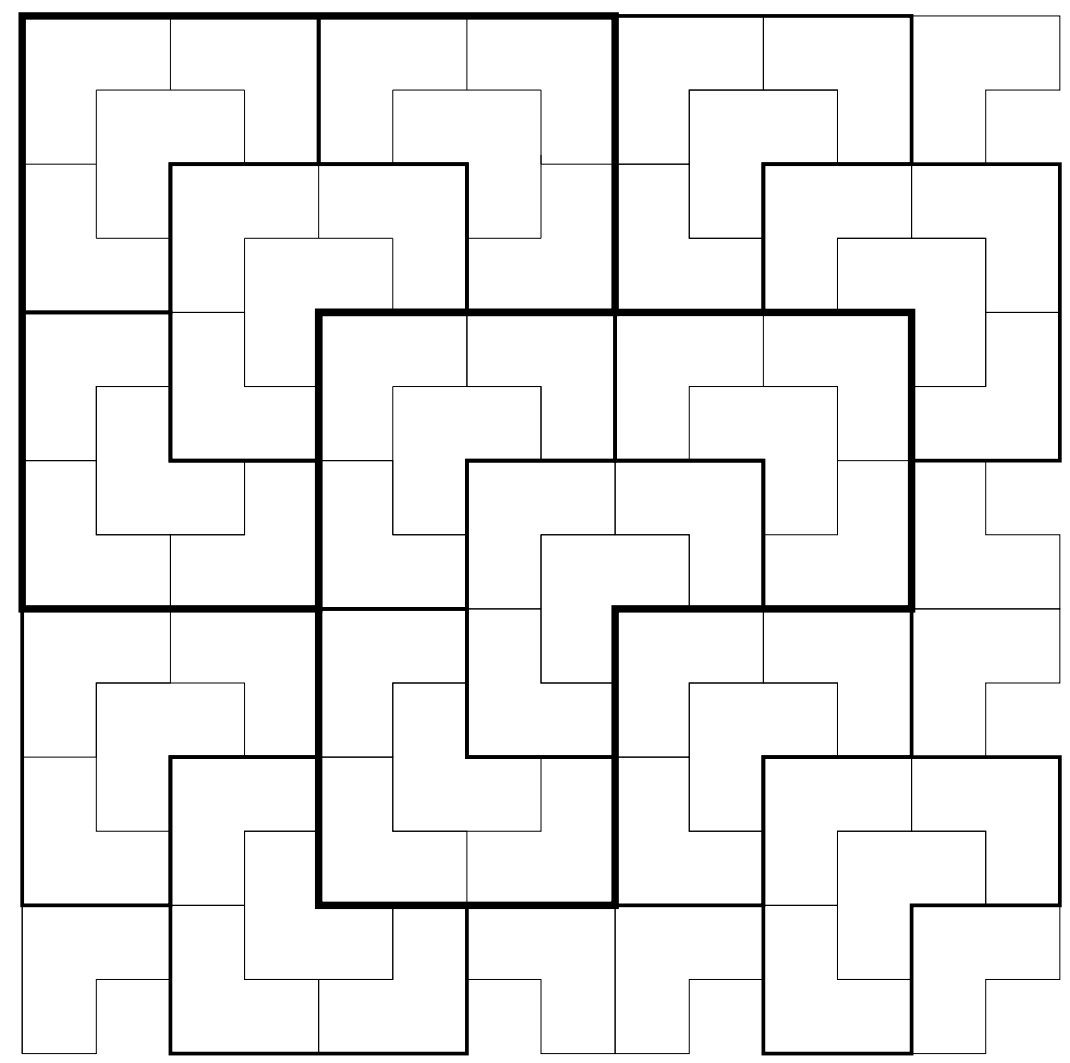

Fig. 2. 63 letters of level 0, 14 letters of level 1 and 2 letters of level 2 in part of a chair tiling 